\title{
Implications of limiting mechanical thrombectomy to patients with emergent large vessel occlusion meeting top tier evidence criteria
}

\author{
Rohini Bhole, ${ }^{1}$ Nitin Goyal, ${ }^{1}$ Katherine Nearing, ${ }^{1}$ Andrey Belayev ${ }^{1,2}$ Vinodh T Doss, ${ }^{1,2}$ \\ Lucas Elijovich, 1,2 Daniel A Hoit, 1,2 Georgios Tsivgoulis, 1,3,4 Andrei V Alexandrov, ${ }^{1}$ \\ Adam S Arthur, ${ }^{1,2}$ Anne W Alexandrov ${ }^{1,4}$
}

\begin{abstract}
${ }^{1}$ Stroke Team, Methodist University Hospital, University of Tennessee Health Science Center, Memphis, Tennessee, USA

${ }^{2}$ Department of Neurosurgery, Semmes-Murphey Brain and Spine Institute, Memphis, Tennessee, USA ${ }^{3}$ Second Department of Neurology, 'Attikon University Hospital', School of Medicine, University of Athens, Athens, Greece

${ }^{4}$ Australian Catholic University, Sydney, Australia
\end{abstract}

\section{Correspondence to} Dr R Bhole, University of Tennessee Health Science Center, Memphis, Department of Neurology, 855 Monroe Avenue, Suite 415, Memphis, TN 38163, USA;

drrohinibhole@gmail.com

Received 10 December 2015 Revised 2 February 2016 Accepted 5 February 2016 Published Online First 1 March 2016

\section{CrossMark}

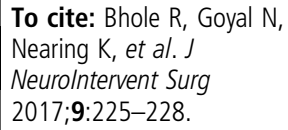

\begin{abstract}
Background Recent guidelines for endovascular management of emergent large vessel occlusion (ELVO) award top tier evidence to the same selective criteria in recent trials. We aimed to understand how guideline adherence would have impacted treatment numbers and outcomes in a cohort of patients from a comprehensive stroke center.

Methods A retrospective observational study was conducted using consecutive emergent endovascular patients. Mechanical thrombectomy (MT) was performed with stent retrievers or large bore clot aspiration catheters. Procedural outcomes were compared between patients meeting, and those failing to meet, top tier evidence criteria.
\end{abstract}

Results 126 patients receiving MT from January 2012 to June 2015 were included (age 31-89 years, National Institutes of Health Stroke Scale (NIHSS) score 2-38); $62(49 \%)$ patients would have been excluded if top tier criteria were upheld: pretreatment NIHSS score $<6(10 \%)$, Alberta Stroke Program Early CT score $<6$ (6.5\%), premorbid modified Rankin Scale (mRS) score $\geq 2(27 \%)$, M2 occlusion (10\%), posterior circulation (32\%), symptom to groin puncture $>360 \mathrm{~min}(58 \%)$. 26 (42\%) subjects had more than one top tier exclusion. Symptomatic intracerebral hemorrhage $(\mathrm{s} \mathrm{ICH})$ and systemic hemorrhage rates were similar between the groups. 3 month mortality was $45 \%$ in those lacking top tier evidence compared with $26 \%$ $(p=0.044)$, and 3 month mRS score $0-2$ was $33 \%$ versus $46 \%$, respectively (NS). After adjusting for potential confounders, top tier treatment was not associated with neurological improvement during hospitalization $(\beta-8.2$; $95 \% \mathrm{Cl}-24.6$ to $-8.2 ; p=0.321), 3$ month mortality (OR=0.38; $95 \% \mathrm{Cl} 0.08$ to 1.41$)$, or 3 month favorable $\mathrm{mRS}(\mathrm{OR}=0.97 ; 95 \% \mathrm{Cl} 0.28$ to 3.35$)$.

Conclusions Our study showed that with strict adherence to top tier evidence criteria, half of patients may not be considered for MT. Our data indicate no increased risk of sICH and a potentially higher mortality that is largely due to treatment of patients with basilar occlusions and those treated at an extended time window. Despite this, good functional recovery is possible, and consideration of MT in patients not meeting top tier evidence criteria may be warranted.

\section{INTRODUCTION}

Recently published guidelines for endovascular treatment of acute ischemic stroke ${ }^{1}$ assign the highest level of evidence to carefully selected patients mirroring the clinical trial inclusion criteria from Multicenter Randomized Clinical trial of Endovascular treatment for Acute ischemic stroke in the Netherlands (MR CLEAN), Endovascular Treatment for Small Core and Proximal Occlusion Ischemic Stroke (ESCAPE), Extending the Time for Thrombolysis in Emergency Neurological Deficits-Intra-Arterial (EXTEND-IA), Solitaire With the Intention For Thrombectomy as Primary Endovascular Treatment (SWIFT PRIME), and Endovascular Revascularization With Solitaire Device Versus Best Medical Therapy in Anterior Circulation Stroke Within 8 Hours (REVASCAT). ${ }^{2-6}$ Despite three previous randomized controlled trials showing no benefit for endovascular treatment, ${ }^{7-9}$ better team coordination, rapid response times, the use of newer mechanical thrombectomy (MT) technologies, and careful patient selection have now demonstrated improved patient outcomes at 3 months in patients treated with MT. Interestingly, none of the recent MT papers provide sufficient data that allow practitioners to fully understand the prevalence of patients meeting top tier evidence criteria for endovascular treatment, and it remains unclear whether MT holds benefit at 3 months for patients outside current guideline recommendations.

Patients with emergent large vessel occlusion (ELVO) are a heterogeneous group requiring rapid and effective revascularization with the likelihood of benefit decreasing over time elapsed from symptom onset. ${ }^{10}$ While exceptions for use of MT in cases not meeting these strict criteria are allowable, it remains unknown whether some centers will exclude treatment of cases not meeting the highest level of evidence requirements, thereby potentially disadvantaging these patients and limiting recovery. ${ }^{11}$ Additionally, concerns about payer support for use of MT in patients not meeting top tier criteria have arisen, given the actions occurring in some states after the publication of earlier negative endovascular trials.

While future clinical trials and post market registries are needed to determine the generalizability of MT to broader ELVO populations, a need for clinicians to make prudent decisions in diverse clinical settings must be acknowledged in patients with significant neurologic disability. We therefore sought 
to compare disability and functional outcomes in patients that underwent MT at our comprehensive stroke center (CSC), according to whether these patients met the top tier criteria for endovascular treatment set forth in the new guidelines.

\section{METHODS}

We conducted a retrospective observational study of consecutive acute ischemic stroke patients registered in our institutional review board approved database that were treated with MT as per the local standard of care at our CSC between January 2012 and June 2015. Patients included in the analysis may have received intravenous (IV) alteplase (recombinant tissue plasminogen activator (tPA)) prior to undergoing MT, and MT methods were not limited to primary deployment of stent retrievers or large bore clot aspiration catheters, consistent with MR CLEAN $^{3}$ study procedures.

As per our institutional policy, patients with a National Institutes of Health Stroke Scale (NIHSS) score of $\geq 6$ or those with lesser scores and cortical or vertebrobasilar symptoms underwent CT angiogram of the head and neck within $12 \mathrm{~h}$ of symptom onset. All CT angiograms were evaluated by a vascular neurologist and neuroendovascular specialist for the presence of ELVO and intermediate to good collateral score to determine eligibility for MT. ${ }^{12}$ Of note, our CSC did not participate in any of the five pivotal MT trials ${ }^{2-6}$ that serve as the foundation for new guideline recommendations. All cases were coded as either meeting or not meeting top tier evidence recommendations based on their conformance to the following criteria presented in the new guidelines:

- Pre-stroke modified Rankin Scale (mRS) score 0-1

- Acute ischemic stroke with receipt of intravenous recombinant IV tPA within $4.5 \mathrm{~h}$ of onset

- Causative occlusion of the internal carotid artery or proximal (M1) middle cerebral artery (MCA)

- Age 18 years or older

- NIHSS score of $\geq 6$

- Alberta Stroke Program Early CT (ASPECT) score of $\geq 6$ and

- Treatment that can be initiated (groin puncture) within $6 \mathrm{~h}$ of symptom onset. ${ }^{1}$

Prospectively collected outcome and process data were used in this retrospective cohort study as the following dependent variables: neurological improvement during hospitalization (defined as the difference between pretreatment and discharge NIHSS scores); symptomatic intracerebral hemorrhage (sICH), defined as per the Safe Implementation of Thrombolysis in Stroke-Monitoring Study (SITS-MOST) definition (parenchymal hematoma type 2 with NIHSS worsening $\geq 4$ points or death within $36 \mathrm{~h}$ of treatment) ${ }^{13}$; serious hemorrhage (defined as life threatening systemic hemorrhage requiring transfusion); time from groin puncture to recanalization; post-recanalization Thrombolysis in Cerebral Infarction grade; endovascular procedural complications; and 90 day mortality and mRS score.

\section{Statistical analyses}

Statistical comparisons were performed between patient subgroups using the $\chi^{2}$ test, Fisher exact test, unpaired Student $t$ test, and the Mann-Whitney $U$ test, based on the level of data analyzed as indicated for dichotomous or continuous variables. Logistic regression was used to evaluate associations between endovascular reperfusion therapies performed in accordance with or against top tier evidence (American Heart Association class $\mathrm{I} /$ level A) recommendations, with both favorable mRS $(0-2)$ at 3 months and death before and after adjusting for potential confounders (variables with imbalances between groups in univariate analyses). Associations are presented as OR with corresponding 95\% CIs. Statistical significance was achieved if $\mathrm{p} \leq 0.05$ in multivariate logistic regression analyses. Finally, we evaluated neurological improvement during hospitalization between the two groups using multiple linear regression analyses. Associations were presented as unstandardized linear regression coefficients $(\beta)$ with corresponding 95\% CIs. The Statistical Package for Social Science (V.11.5 for Windows; SPSS Inc, Chicago, Illinois, USA) was used for statistical analyses.

\section{RESULTS}

A total of 126 ELVO patients undergoing MT were included in the analysis. Sample characteristics are presented in table 1. Our study population consisted of 64 cases that fulfilled top tier evi-

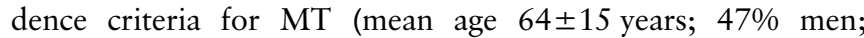
median pretreatment NIHSS $_{\text {score }} 16$ (IQR 14-18)), and 62 $(49 \%)$ cases that did not meet top tier MT evidence criteria (mean age $62 \pm 13$ years; $52 \%$ men; median pretreatment NIHSS score 14 (IQR 7-18) (NS). Characteristics not meeting top tier evidence for MT in the comparison group included 6 $(10 \%)$ patients with a pretreatment NIHSS score of $<6,4$ (6.5\%) patients with an ASPECT score <6, 17 (27\%) patients with a premorbid mRS score $\geq 2,6(10 \%)$ patients with M2 occlusions, $20(32 \%)$ patients with posterior circulation occlusions, and $36(58 \%)$ patients with symptom to groin puncture time $>6 \mathrm{~h} ; 26(42 \%)$ subjects had two or more characteristics removing them from the top tier evidence group.

IV tPA treatment was given to $92 \%$ of cases meeting top tier criteria for MT, whereas only 40\% $(\mathrm{p}<0.001)$ of non-top tier cases received IV tPA, primarily due to late arrival after symptom onset. Top tier cases also had shorter symptom onset to groin puncture times compared with those not meeting top tier evidence criteria (234 min (IQR 177-291) vs $381 \mathrm{~min}$ (IQR $268-454)$, respectively; $\mathrm{p}<0.001$ ) but the median door to groin puncture times between the two groups were not significantly different (151 min (IQR 115-190) vs 149 min IQR (115-237), respectively; $\mathrm{p}=0.628)$.

In our cohort, a combination of distal aspiration and stent triever was used as the most common technique (sometimes called the 'solumbra' technique) in 47 cases (37\%). The second most commonly employed technique was the ADAPT technique, and this was used in 42 cases (33\%). Stent trievers without distal aspiration were used in 27 cases (21\%). The remaining few cases were mostly cervical carotid occlusions in which acute angioplasty and stenting was employed.

Cases not meeting top tier evidence criteria were also more commonly intubated prior to MT for problems including respiratory insufficiency, stroke severity, and/or medical comorbidities contributing to decline in mental status (34\% intubated; $n=21$ patients of whom 13 were basilar artery (BA) occlusions) compared with top tier evidence cases (16\%; $\mathrm{p}=0.020)$. Median ASPECT scores were also higher in cases meeting top tier criteria for MT compared with others (10 (IQR $10-10)$ vs 9 (IQR 8-10), respectively; $p=0.001$ ). Additionally, cases not meeting top tier MT evidence included 20 (32\%) BA occlusion patients, and of these, 11 (55\%) died while $5(25 \%)$ achieved mRS $\leq 2$ at the 3 month follow-up.

Rates for sICH were $8 \%$ in each group, and serious hemorrhage rates were similar at $8 \%$ in top tier MT evidence cases compared with $10 \%$ in other cases $(p=0.731)$. Top tier MT evidence cases achieved greater neurologic improvement during hospitalization (10 points (IQR 6-14)) compared with other cases (5 points (IQR $1-10) ; \mathrm{p}=0.006)$ in initial univariate 
Table 1 Baseline characteristics of the study groups

\begin{tabular}{|c|c|c|c|}
\hline Variable & $\begin{array}{l}\text { Top tier MT evidence } \\
\text { cases }(n=64)^{*}\end{array}$ & $\begin{array}{l}\text { Cases not meeting top tier } \\
\text { evidence for MT }(n=62) t\end{array}$ & p Value \\
\hline \multicolumn{4}{|l|}{ Demographic characteristics } \\
\hline Age (years) $($ mean $\pm S D)$ & $64 \pm 15$ & $62 \pm 13$ & 0.473 \\
\hline Male gender (\%) & 47 & 52 & 0.595 \\
\hline Race $(\%)$ & & & 0.991 \\
\hline White & 47 & 47 & \\
\hline Black & 53 & 53 & \\
\hline \multicolumn{4}{|l|}{ Medical history (\%) } \\
\hline Hypertension & 72 & 81 & 0.248 \\
\hline Diabetes mellitus & 38 & 37 & 0.908 \\
\hline Hypercholesterolemia & 38 & 48 & 0.217 \\
\hline Atrial fibrillation & 33 & 29 & 0.646 \\
\hline Smoking & 30 & 32 & 0.848 \\
\hline Congestive heart failure & 11 & 16 & 0.433 \\
\hline Coronary artery disease & 23 & 29 & 0.475 \\
\hline \multicolumn{4}{|l|}{ Clinical and imaging characteristics } \\
\hline SBP $(\mathrm{mm} \mathrm{Hg})($ mean \pm SD) & $155 \pm 25$ & $160 \pm 32$ & 0.323 \\
\hline $\mathrm{DBP}(\mathrm{mm} \mathrm{Hg})(\mathrm{mean} \pm \mathrm{SD})$ & $88 \pm 18$ & $92 \pm 22$ & 0.257 \\
\hline Glucose (mg/dL) (median, IQR) & $125(95-158)$ & $122(104-167)$ & 0.791 \\
\hline Admission NIHSS score (median, IQR) & $16(14-18)$ & $14(7-18)$ & 0.427 \\
\hline ASPECT score (median, IQR) & $10(10-10)$ & $9(8-10)$ & 0.001 \\
\hline Intravenous thrombolysis (\%) & 92 & 40 & $<0.001$ \\
\hline Onset to tPA time (min) (median, IQR) & $117(89-148)$ & $140(102-207)$ & 0.108 \\
\hline Good collateral score (\%) & 45 & 40 & 0.510 \\
\hline Intubation prior or during the procedure (\%) & 16 & 34 & 0.020 \\
\hline Symptom onset to groin puncture time (min) (median, IQR) & $234(177-291)$ & $381(268-454)$ & $<0.001$ \\
\hline Door to groin puncture (min) (median, IQR) & $151(115-190)$ & $149(115-237)$ & 0.628 \\
\hline
\end{tabular}

analyses. Mortality was significantly lower in top tier evidence cases at $26 \%$ compared with $45 \%$ in other cases $(p=0.044)$, whereas favorable outcome $(\mathrm{mRS} \leq 2)$ at 3 months was similar at $46 \%$ versus $33 \%$, respectively $(p=0.158)$. After adjusting for potential imbalances between the two groups, including baseline ASPECT score, IV tPA pretreatment, intubation, and time elapsed from symptom onset to groin puncture, associations between endovascular MT in top tier evidence cases and others did not reach statistical significance in multivariate analysis (table 3).

\section{DISCUSSION}

Our study showed that despite lacking top tier evidence for MT, $33 \%$ of cases treated outside these recommendations attained an mRS score of $\leq 2$ by 3 months, and that nearly half of our MT cases would have been denied MT if top tier evidence criteria

Table 2 Outcomes of the study groups in initial univariate analyses

\begin{tabular}{|c|c|c|c|}
\hline Outcome variable & $\begin{array}{l}\text { Top tier MT evidence } \\
\text { cases }(n=64)^{*}\end{array}$ & $\begin{array}{l}\text { Cases not meeting top tier } \\
\text { evidence for MT }(n=62) t\end{array}$ & p Value \\
\hline Groin puncture to recanalization time (min) (median, IQR) & $54(43-83)$ & $42(26-60)$ & 0.008 \\
\hline Final $\mathrm{TICI} \mathrm{Ilb} / \mathrm{III}(\%)$ & 69 & 67 & 0.854 \\
\hline $\begin{array}{l}\text { Reduction in NIHSS score at discharge (from pretreatment NIHSS score) } \\
\text { (median, IQR) }\end{array}$ & $10(6-14)$ & $5(1-10)$ & 0.006 \\
\hline $\mathrm{sICH}(\%)$ & 8 & 8 & 0.979 \\
\hline Serious hemorrhagic complications (\%) & 8 & 10 & 0.731 \\
\hline Surgical treatment following endovascular therapy (\%) & 6 & 7 & 0.944 \\
\hline Mortality at 3 months (\%) & 26 & 45 & 0.044 \\
\hline mRS $0-2$ at 3 months (\%) & 46 & 33 & 0.158 \\
\hline
\end{tabular}

*Fulfilling criteria American Heart Association class I level A recommendations.

†Not fulfilling criteria American Heart Association class I level A recommendations.

mRS, modified Rankin Scale; MT, mechanical thrombectomy; NIHSS, National Institutes of Health Stroke Scale; TICl, Thrombolysis in Cerebral Infarction; sICH, symptomatic intracerebral hemorrhage. 
Table 3 Associations between endovascular reperfusion therapies performed according to American Heart Association class I level A recommendations (reference group includes patients treated with endovascular reperfusion therapies outside American Heart Association class I level A recommendations) with early outcomes on multivariate analyses

\begin{tabular}{|c|c|c|c|}
\hline $\begin{array}{l}\text { Multivariate } \\
\text { analysis* }\end{array}$ & Early outcome & Association & $\mathrm{p}$ Value \\
\hline Logistic regression & 3 month mortality & $\begin{array}{l}\text { OR: } 0.38 \text { (0.08 to } \\
1.41)\end{array}$ & 0.135 \\
\hline Logistic regression & $\begin{array}{l}3 \text { month favorable functional } \\
\text { outcomet }\end{array}$ & $\begin{array}{l}\text { OR: } 0.97 \text { ( } 0.28 \text { to } \\
3.35)\end{array}$ & 0.963 \\
\hline Linear regression & $\begin{array}{l}\text { Neurological improvement } \\
\text { during hospitalization } \neq\end{array}$ & $\begin{array}{l}\beta:-8.2(-24.6 \\
\text { to }-8.2)\end{array}$ & 0.321 \\
\hline
\end{tabular}

*Adjusting for Alberta Stroke Program Early CT score, intravenous thrombolysis, intubation during the procedure, and elapsed time from symptom onset to groin puncture.

tModified Rankin Scale score of $0-2$.

¥Quantified using the difference between admission and discharge NIHSS scores.

$\beta$, unstandardized linear regression coefficient.

NIHSS, National Institutes of Health Stroke Scale.

were upheld in clinical practice. Additionally, our data indicated no increased risk of sICH or serious hemorrhagic complications in the group that lacked top tier evidence for MT, demonstrating that a sizeable percentage of patients not meeting top tier criteria may still have a good outcome with MT.

Our data showed that even though the non-top tier group was treated later from symptom onset, both groups did not differ significantly by door to groin puncture times. It is likely that the top tier inclusion criterion of $6 \mathrm{~h}$ played a role in delayed puncture times from symptom onset in the non-top tier group and not the door times. Despite guidelines ${ }^{1}$ endorsing stent trievers as the technology of choice for thrombectomy, our data showed that in clinical practice, aspiration and a combination of technologies (aspiration and stent trievers) played a greater role in the treatment of ELVO.

Overall, our findings further support dependency of functional recovery on time to intervention, with favorable outcomes at 3 month achieved by 13\% fewer patients when time to groin puncture was delayed over $360 \mathrm{~min}$. Of note, $15 \%$ fewer patients recovered in the Interventional Management of Stroke (IMS) 3 trial with angiographic reperfusion delayed to beyond 360 min. ${ }^{10}$ However, in the ESCAPE trial, ${ }^{3} 49$ patients underwent randomization $6 \mathrm{~h}$ or more after stroke onset, with the direction of effect in this subgroup favoring intervention for mRS 0-2 at 90 days. Good collateral scores are able to predict smaller infarct volumes and better clinical outcome in patients undergoing MT. ${ }^{12}$ We conclude that careful selection of patients for MT with good collaterals at an extended window may offer benefit with very little risk, and therefore advocate for future research in patients outside the $6 \mathrm{~h}$ window. Post market registries rather than future randomized controlled trials may be more feasible in certain patient subgroups for examining the value of MT in patients with:

1. Low NIHSS score $<6$

2. ASPECT score $<6$

3. Pre-stroke mRS score $>1$

4. Occlusion of the M2 or M3 portion of the MCAs, anterior cerebral arteries, vertebral arteries, BAs, or posterior cerebral arteries and

5. Treatment beyond $6 \mathrm{~h}$ from symptom onset for patients with acute ischemic stroke who have causative occlusion of the internal carotid artery or proximal MCA (M1). ${ }^{1}$
We attribute the higher mortality in patients lacking top tier evidence for MT to the high representation of BA occlusions $(32 \%)$ and those treated at an extended time window. BA occlusion has an invariably poor outcome if recanalization is not achieved early. ${ }^{14} 15$ Arnold et al showed that lower NIHSS and recanalization of BA occlusions predict favorable outcomes after intra-arterial thrombolysis. ${ }^{14}$ We treated basilar occlusions beyond the $6 \mathrm{~h}$ window with overall mortality similar to that demonstrated by previous investigators, ranging between $43 \%$ and $45 \% .^{14} 16$

Our study has limitations, including its retrospective observational design with lack of concurrent controls, a relatively small sample size, single site and team management, and selection bias towards more disabled patients with a higher ASPECT score and good collateral scores. However, as non-participants in any of the recent endovascular trials from which the new MT guidelines were derived, ${ }^{2-6}$ our study offers a first look at outcomes of patients treated routinely in a CSC. A multicenter registry should be established to build knowledge of the value of MT in patients lacking top tier evidence of favorable outcome. Newer thrombectomy techniques are being rapidly developed, and additional outcome data in these patients are needed to power future clinical trials. ${ }^{17}$

In conclusion, our data support careful use of MT in patients that do not meet current top tier evidence for favorable outcome, and continued study of the efficacy and safety of current and evolving technologies to manage ELVO in broader patient populations. The role of MT could be significantly expanded if patients are selected based on risk of severe disability and favorable parenchymal/collateral imaging findings, instead of time and occlusion location.

Contributors RB: contribution to conception of the work, data acquisition and analysis, and drafting and revising the manuscript. $N G, K N$, and $A B$ : data acquisition. VTD, LE, DAH, and ASA: critical revision of the manuscript and approval of the final version. AVA and GT: substantial contribution to conception of the work, analysis and interpretation of the data, critically revising manuscript, and approval of the final version. AWA: critical revision of the manuscript for intellectual content and approval of the final version.

Competing interests ASA: consultant for Codman, Medtronic, Microvention, Penumbra, Sequent, Siemens, Silk Road, and Stryker; investor for Valor Medical; and research support from Codman, Penumbra, Sequent, and Siemens.

Ethics approval The study was approved by the institutional review board of the University of Tennessee Health Science Center.

Provenance and peer review Not commissioned; externally peer reviewed.

Data sharing statement All authors are willing to share the database with authors interested in collaborative research.

\section{REFERENCES}

1 Powers WJ, Derdeyn CP, Biller J, et al. 2015 AHA/ASA focused update of the 2013 guidelines for the early management of patients with acute ischemic stroke regarding endovascular treatment: a guideline for healthcare professionals from the American Heart Association/American Stroke Association. Stroke 2015;46:3020-35.

2 Berkhemer OA, Fransen PS, Beumer D, et al. A randomized trial of intraarterial treatment for acute ischemic stroke. N Engl J Med 2015;372:11-20.

3 Goyal M, Demchuk AM, Menon BK, et al. Randomized assessment of rapid endovascular treatment of ischemic stroke. N Engl J Med 2015;372:1019-30.

4 Campbell BC, Mitchell PJ, Kleinig TJ, et al. Endovascular therapy for ischemic stroke with perfusion-imaging selection. N Engl J Med 2015;372:1009-18.

5 Saver JL, Goyal M, Bonafe A, et al. Stent-retriever thrombectomy after intravenous t-PA vs. t-PA alone in stroke. N Engl J Med 2015;372:2285-95.

6 Jovin TG, Chamorro A, Cobo $E$, et al. Thrombectomy within 8 hours after symptom onset in ischemic stroke. N Engl J Med 2015;372:2296-306.

7 Kidwell CS, Jahan R, Gornbein J, et al. A trial of imaging selection and endovascular treatment for ischemic stroke. N Engl J Med 2013;368:914-23.

8 Broderick JP, Palesch YY, Demchuk AM, et al. Endovascular therapy after intravenous t-PA versus t-PA alone for stroke. N Engl J Med 2013;368:893-903.

9 Ciccone A, Valvassori L, Investigators SE. Endovascular treatment for acute ischemic stroke. N Engl J Med 2013;368:2433-4. 
10 Khatri P, Yeatts SD, Mazighi M, et al. Time to angiographic reperfusion and clinical outcome after acute ischaemic stroke: an analysis of data from the Interventional Management of Stroke (IMS iii) phase 3 trial. Lancet Neurol 2014;13:567-74.

11 Fiorella D, Mocco J, Arthur AS. Too much guidance. J Neurolntervent Surg 2015;7:626-7.

12 Elijovich L, Goyal N, Mainali S, et al. CTA collateral score predicts infarct volume and clinical outcome after endovascular therapy for acute ischemic stroke: a retrospective chart review. J Neurointervent Surg doi.org/10.1136/neurintsurg2015-011731

13 Wahlgren N, Ahmed N, Dávalos A. Thrombolysis with alteplase for acute ischaemic stroke in the Safe Implementation of Thrombolysis in Stroke-Monitoring Study (SITS-MOST): an observational study. Lancet 2007;369:275-82.
14 Arnold M, Nedeltchev K, Schroth G, et al. Clinical and radiological predictors of recanalisation and outcome of 40 patients with acute basilar artery occlusion treated with intra-arterial thrombolysis. J Neurol Neurosurg Psychiatr 2004;75:857-62.

15 Kumar G, Shahripour RB, Alexandrov AV. Recanalization of acute basilar artery occlusion improves outcomes: a meta-analysis. J Neurolntervent Surg 2015;7: 868-74.

16 Schulte-Altedorneburg G, Hamann GF, Mull M, et al. Outcome of acute vertebrobasilar occlusions treated with intra-arterial fibrinolysis in 180 patients. AJNR Am J Neuroradiol 2006;27:2042-7.

17 Zaidat 00, Castonguay AC, Gupta $R$, et al. North American solitaire stent retriever acute stroke registry: post-marketing revascularization and clinical outcome results. J Neurointerv Surg 2014;6:584-8. 